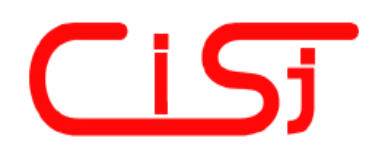

\title{
SMART SILICON SENSORS BASED ON VERTICAL HALL EFFECT DEVICES
}

\author{
Konstantin Dimitrov, Chavdar Roumenin \\ Institute of control and system research at the Bulgarian academy of sciences, \\ 1113 Sofia, BULGARIA; P.O. Box 79 \\ tel/fax: (+359 2) 8737822 \\ kdimitrov@icsr.bas.bg, roumenin@bas.bg \\ www.icsr.bas.bg
}

\begin{abstract}
Future integrated systems will benefit significantly from the progress in batch manufactured silicon sensors and signal processing techniques. Silicon technologies make possible to produce sensing microdevices combining maximal sensitivity, high accuracy and minimal design complexity. Smart sensors on the base of Vertical vector Hall effect devices offer a number of advantages including reducing mass, volume and power consumption; greater redundancy of system functions and simpler architecture. In view of these characteristics, it can be expected that such smart sensors will be used extensively wide if an adequate solution is found to reduce the design cost and simplify the electrical interface. Consequently, cost effective microsystems including vector magnetic sensors, circuits and eventually actuators can be fabricated.

This paper presents a new approach in the field of signal processing for magnetic field sensors based on vertical Hall elements. A design example is illustrated specific problems and solutions associated with data converters and signal-processing functions for smart sensors.
\end{abstract}

Keywords: Vertical Hall effect devices, Signal Processing, Smart Sensors.

\section{INTRODUCTION}

Sensors are not always suitable for applications in data-acquisition and sensor systems. There are two reasons for this: appropriate standards for the electrical output signals; and that signals show undesirable disadvantages such as offset, nonlinearity (NL), cross-sensitivity (CS) etc. To apply a magnetic sensor system designers must be thoroughly familiar with its performance. Without of this knowledge they will be unable to build a manageable system of replace sensors with standard output formats.

Traditional interface function, such as analogue sensor signal conditioning, analogue to digital (A/D) conversion and the generation of reference signals, take place in smart sensors.

Smart sensors include on the same micro chip the sensing element and the interface circuit, in order to perform a suitable of the sensor signals before the transmission to the rest of the system [1-6]. There are two basic different ways to be realized smart sensors. At the first one - sensors can be fabricated directly on the silicon substrate used for the integrated circuits and the second - sensors have to be assembled together with the interface circuit in a multi-chip module. Both approaches allow batch manufacturing of either - the integrated circuits and the sensing elements. Smart sensors typically require specific signal processing functions to match characteristics of both sensor and system.

This paper contains our recent activity in the field of signal processing for smart sensors in the base of vertical Hall microstructures. Part of new results is already presented [1]. A few design examples are reported to illustrate specific problems and solutions associated with data converters and signal processing functions for smart sensors.

\section{DATA CONVERTERS}

In sensor systems, after the preliminary signal processing performed by the front-end circuitry, in the most cases is necessary to convert the signals in the digital domain. This section discusses some practical implementations of analog to digital (A/D) converters for smart sensor systems based on various conversion techniques. When low power consumption and fast conversion time are required, 
successive approximation A/D converters are widely used.

\subsection{2-D Hall magnetic sensor in the base of incremental A/D converter}

Silicon Hall magnetic sensors are generally compatible with standard IC technologies. Fig. 1 shows a functionally integrated 2-D silicon vector microsystem $[7,8]$. It consist two triple parallel-field Hall sensors replaced each other on the $90^{\circ}$ in plane $x-y[8-10]$.

The device contains a central square current contact $\mathrm{C}_{0}$ and one contact on each side $\mathrm{C}_{1}-\mathrm{C}_{4}$ symmetrically situated around $\mathrm{C}_{0}$. The two differential outputs generate $V_{H}\left(\boldsymbol{B}_{x}\right)$ and $V_{H}\left(\boldsymbol{B}_{y}\right)$ Hall voltages, proportionally to the $x$ - and $y$-components of the magnetic field.

The measurement of the cross-sensitivity at current $I_{\mathrm{C} 0}=$ const., after nullification of the two offsets we achieved, using the following procedure. The first step is an experimental determination of the two channels magnetosensitivities at Hall-voltage mode $V_{H}\left(\boldsymbol{B}_{x}\right)$ and $V_{H}\left(\boldsymbol{B}_{y}\right)$ of operation. The next step is applying a homogeneous variable field $B$ parallel to one of the axis, for example $B_{x}$-axis. The other output (parasitic) signal from the $y$ - channel is registered simultaneously. Then the dependence of the relative change of the parasitic signal from the $y$ direction $V_{H y}\left(\boldsymbol{B}_{x}\right) / V_{H y}\left(\boldsymbol{B}_{y}\right)$ is plotted in \% versus the magnetic induction $B_{x}$ applied to the $x$-axis. The procedure described above is repeated for the remaining directions. In our case, because of the device symmetry, the relation $S_{x y}=S_{y x}$ and $S_{x z}=S_{y z}$ between respective cross-sensitivity is valid.

On Fig. 2 is presented the cross-sensitivity of the 2-D microsystem. The CS is close to a square function of the induction $B$. This proves the dominant role of the geometrical magnetoresistance in CS.

On Fig. 3 is presented vector Hall magnetic sensor and a biasing circuitry for measuring the internal noise.

The measurement set-up on Fig. 3 included: Hall sensor both with current biasing generator realized through accumulator, placed in a metal shielded box; Li-Ion rechargeable batteries which is also placed in a metal shielded box; wide-band low-noise operational amplifier with gain amplification coefficient non smaller than $10^{4}$, which is also biasing by an accumulator; oscilloscope connected with the amplifier's output to be observed output signal behavior; digital spectral analyzer with a fast Furie transformation for the noise spectrum computation of the output sensor signal. A special attention could be paid on the sensor screening from electromagnetic disturbances, on amplifier and on the biasing circuits. The set-up used for the noise measurement is especially created for this goal instruments on the base of digital spectral analyzer Philips PM 3360.

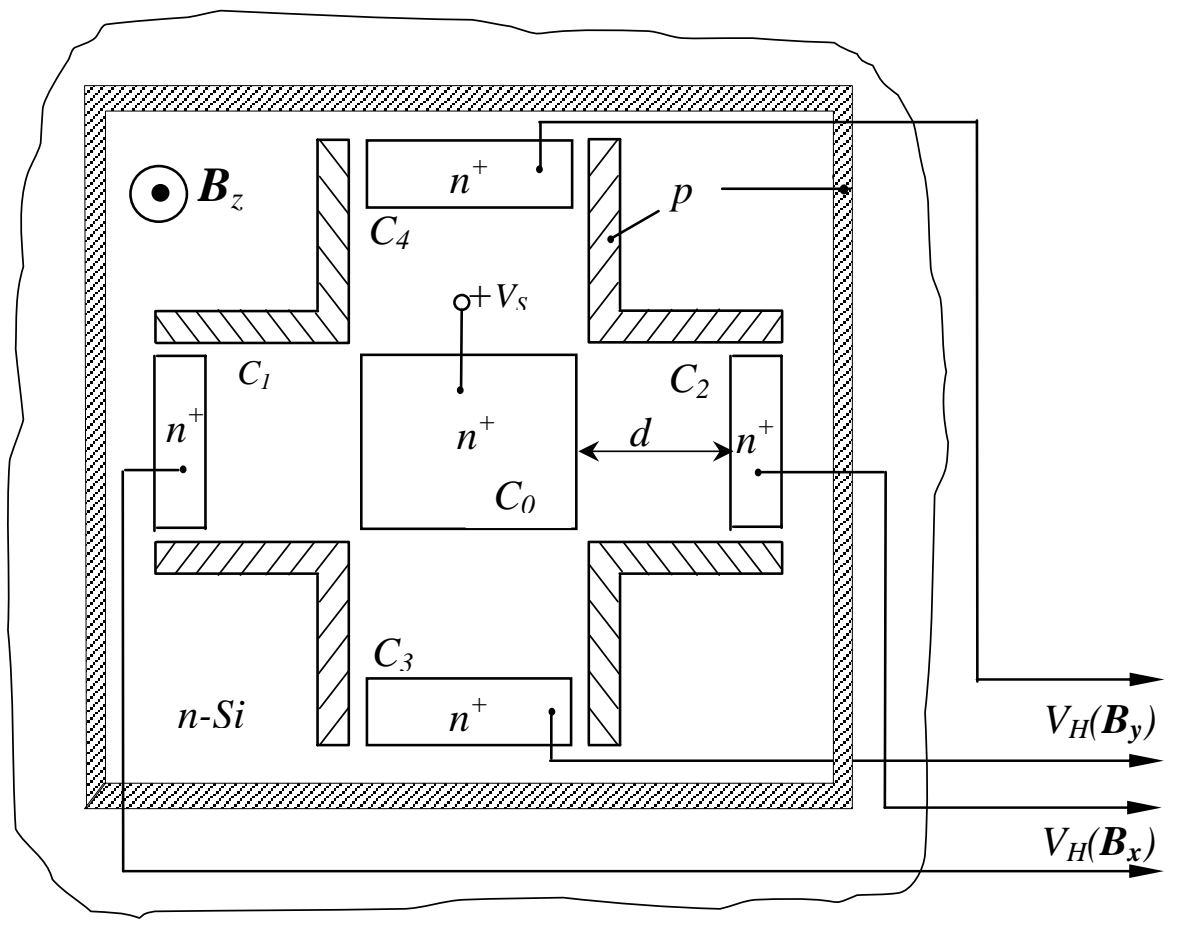

Fig. 1 - 2-D magnetic microsystem for the in-plane $B_{x}$ and $B_{y}$ magnetic field components. 


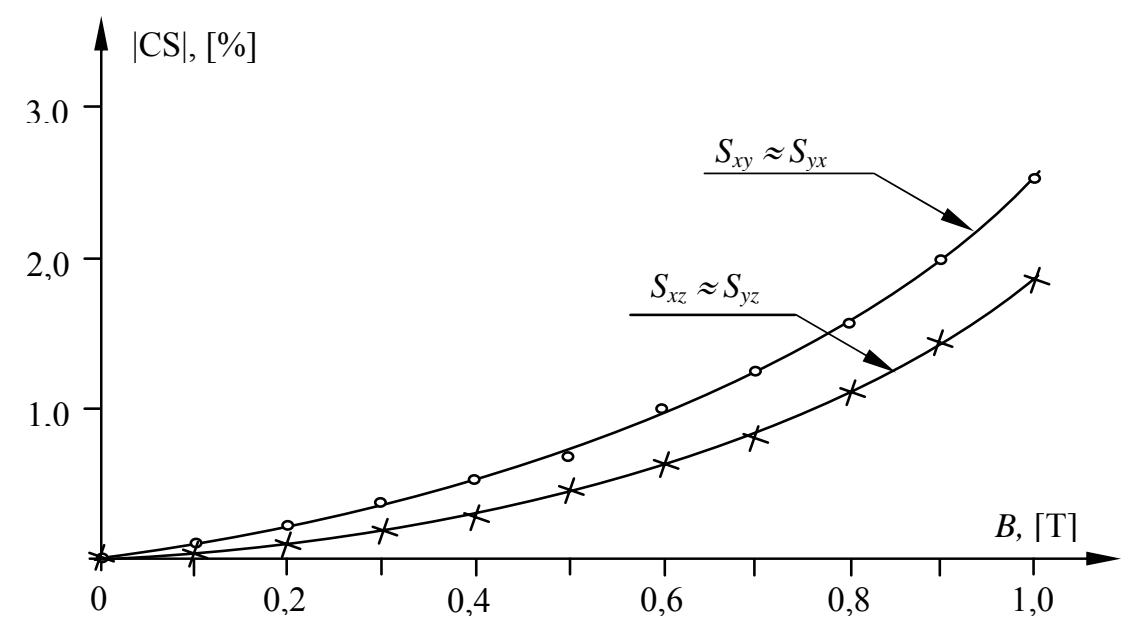

Fig. 2 - The cross-sensitivity between the $x$ - and $y$ - channels for $I_{\mathrm{C} 0}=10 \mathrm{~mA}, T=300 \mathrm{~K}$.

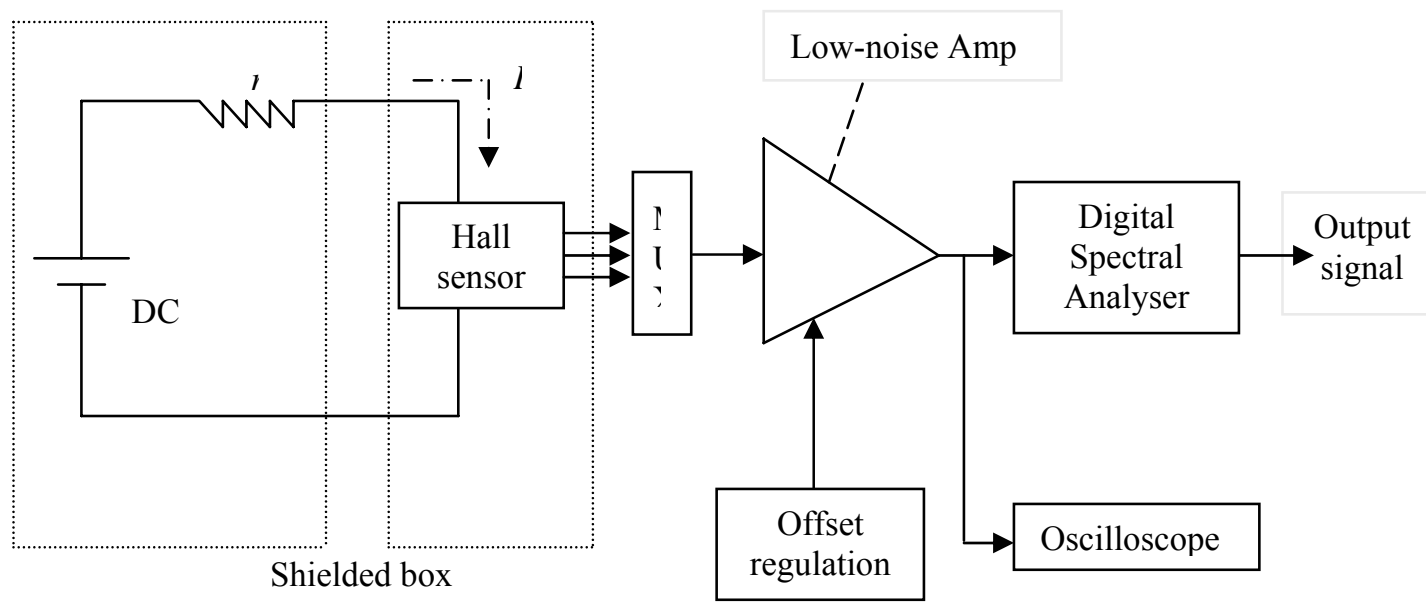

Fig. 3 - Hall magnetic sensor and a biasing circuitry for noise measurement.

In Fig. 4 is shown measured noise power spectral density for one of the sensor channels, of the microsystem of Fig. 1, in function of frequency $f$ at magnetic field $B=0$. There is established that the behavior of this important sensor parameter at low frequencies $f \leq 10^{3}-10^{4} \mathrm{~Hz}$ doesn't differentiate from the expected one, i.e. the $1 / f$ noise (Flicker noise).

The grow up of the noise level with the increasing of the supply current $I_{0}$ is due to the increasing role of the carriers scattering, because of the higher velocity in the electric field. Among them we will consider the 2-D magnetic sensor system for angular measurements, whose scheme is shown in Fig. 5 [5-7, 10-12].

Vector 2-D Hall magnetometer [8, 11, 12] together with the front-end circuitry deliver two differential voltages proportional to $\boldsymbol{B}_{\boldsymbol{x}}$ and $\boldsymbol{B}_{\boldsymbol{y}}$ components of the magnetic field produced by a permanent magnet placed on top of the chip. The sensor geometry and the read out circuit have been optimized to reduce offset, temperature and noise parametric dependences and to increase sensitivity of the system as all [7 - 9]. Since the resulting front-end output voltages are in the order of few tens of $\mathrm{mV}$ only, a couple of operational amplifiers are required to achieve a reasonably large signal level at the input of the subsequent $\mathrm{A} / \mathrm{D}$ converter $[4,6,7]$.

Differential output voltages of the operational amplifier (OA) represent alternatively the input or the reference signal of an incremental $A / D$ converter, depending on the quadrant of the magnetic field angle $\alpha$. The correct value of $\alpha$ is determinate by a finite state machine in the digital section [4]. When quadrant is determined, the bitstream delivered by the A/D converter is decimated by a counter, producing an output word $N$ proportional to the ratio between the magnetic field components. Finally, a look-up table transforms $N$ into the magnetic field angle using a Gray code representation.

In order to avoid abnormal operation of the vector probe due to the absence of the permanent magnet, in the system is included two window comparators, which check the magnetic field strength and deliver to the digital section a suitable status bit.

The measured output digital word as a function of the magnetic field angle is shown in Fig. 5. 


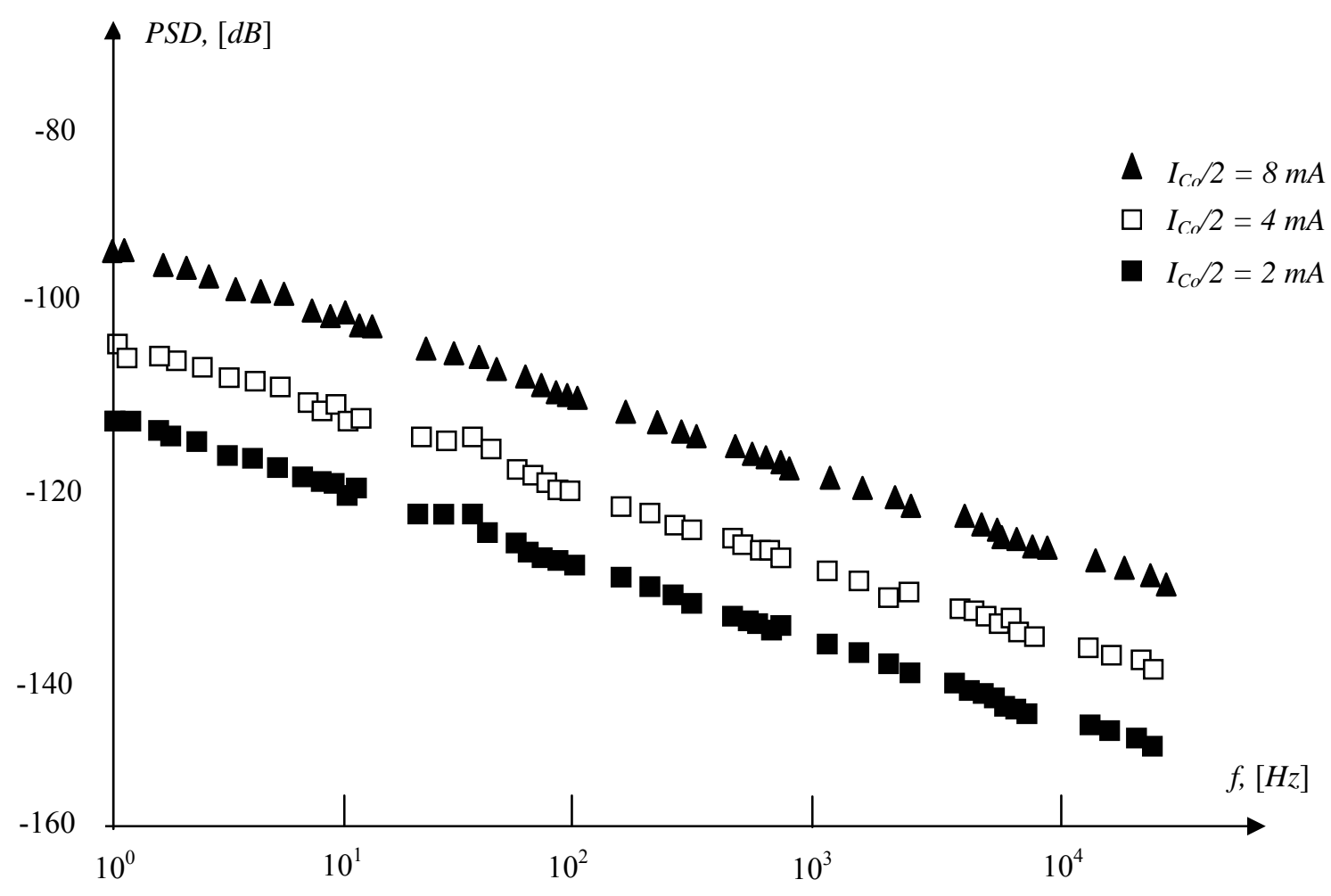

Fig. 4 - Noise power spectral density for one of the sensor channels, as a parameter is chosen the supply current $I_{\mathrm{C} 0} / 2$ at temperature $T=300 \mathrm{~K}$.

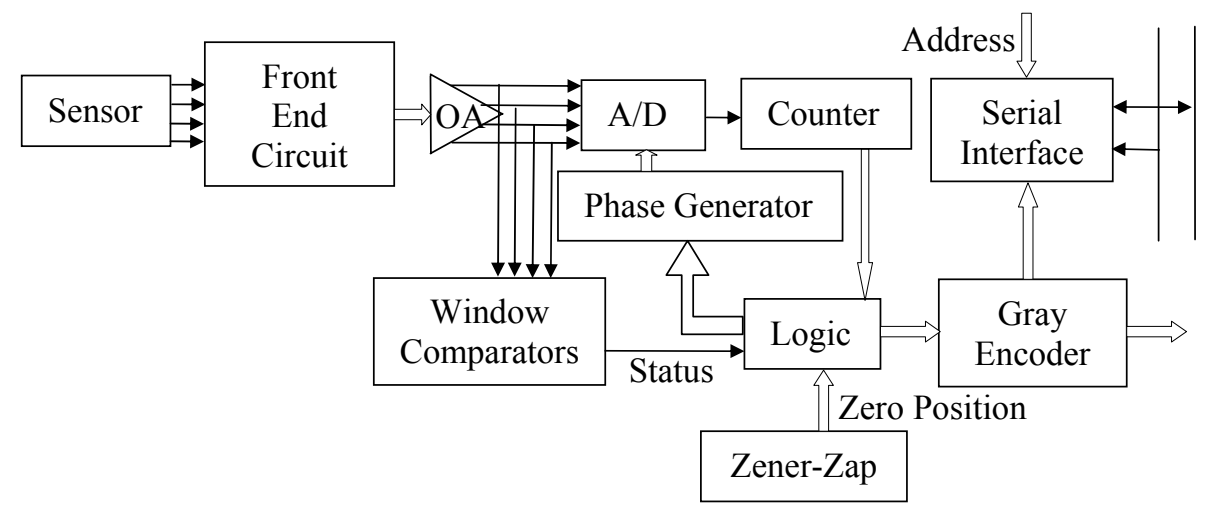

Fig. 5 - Block diagram of the rotary switch interface circuit.

\subsection{Successive approximation A/D converter based on 3-D Hall magnetic sensor}

Several working environments are polluted by strong low-frequency magnetic field. In order to guarantee the health of the workers, it is essential to monitor the exact integral exposure of each worker to a particular level of filed with magnetodosimeter.

The level of magnetic field which is known to be dangerous for human beings can be detected by silicon magnetic sensors fabricated in conventional CMOS technologies. However, the bias current required to be achieved the desired sensitivity.
The magnetic sensor used to detect $\boldsymbol{B}_{\boldsymbol{x}}, \boldsymbol{B}_{\boldsymbol{y}}$ and $\boldsymbol{B}_{\boldsymbol{z}}$ is a 3-D silicon vector sensor, completely realizable with the methods of IC technology, Fig. 6. The device contains a central current ohmic contact $\mathrm{C}_{1}$ and one more contact on each side $\mathrm{C}_{2}$ and $\mathrm{C}_{3}$ symmetrically placed around $\mathrm{C}_{1}$. The Hall terminals are placed in following way: $\mathrm{H}_{1}$ and $\mathrm{H}_{2}$ - in the zone between $\mathrm{C}_{1}$ and $\mathrm{C}_{2} ; \mathrm{H}_{3}$ and $\mathrm{H}_{4}$ - at either side of contact $\mathrm{C}_{1}$, and $\mathrm{H}_{5}$ and $\mathrm{H}_{6}$ - in the zone between $\mathrm{C}_{1}$ and $\mathrm{C}_{3}$. Also the contacts $\mathrm{H}_{1}$ and $\mathrm{H}_{6}$ and, respectively, $\mathrm{H}_{2}$ and $\mathrm{H}_{5}$ are cross-coupled, which increase the signal for the component $\boldsymbol{B}_{\boldsymbol{z}}$ and neutralizes the influence of the other fields $\boldsymbol{B}_{x}$ and $\boldsymbol{B}_{\boldsymbol{y}}$ in this output channel [10,13 - 15]. 
Fig. 7 contains the results for the parameter CS. In accordance with the experimental results a reduction of CS of about $45 \%$ with respect to the Hall voltage mode of operation using an amperometric circuit only is achieved.

The measured noise power spectral density of the vector sensor for one of the channels is shown in
Fig. 8. For the other two channels the dependencies are the same. As can be seen, in the range $1-1000$ $\mathrm{Hz}$ the internal noise is of the type $1 / f$, and as the current $I_{C 1}$ increases the noise increases too.

The block-diagram of the integrated magnetodosimeter is shown in Fig. 9.

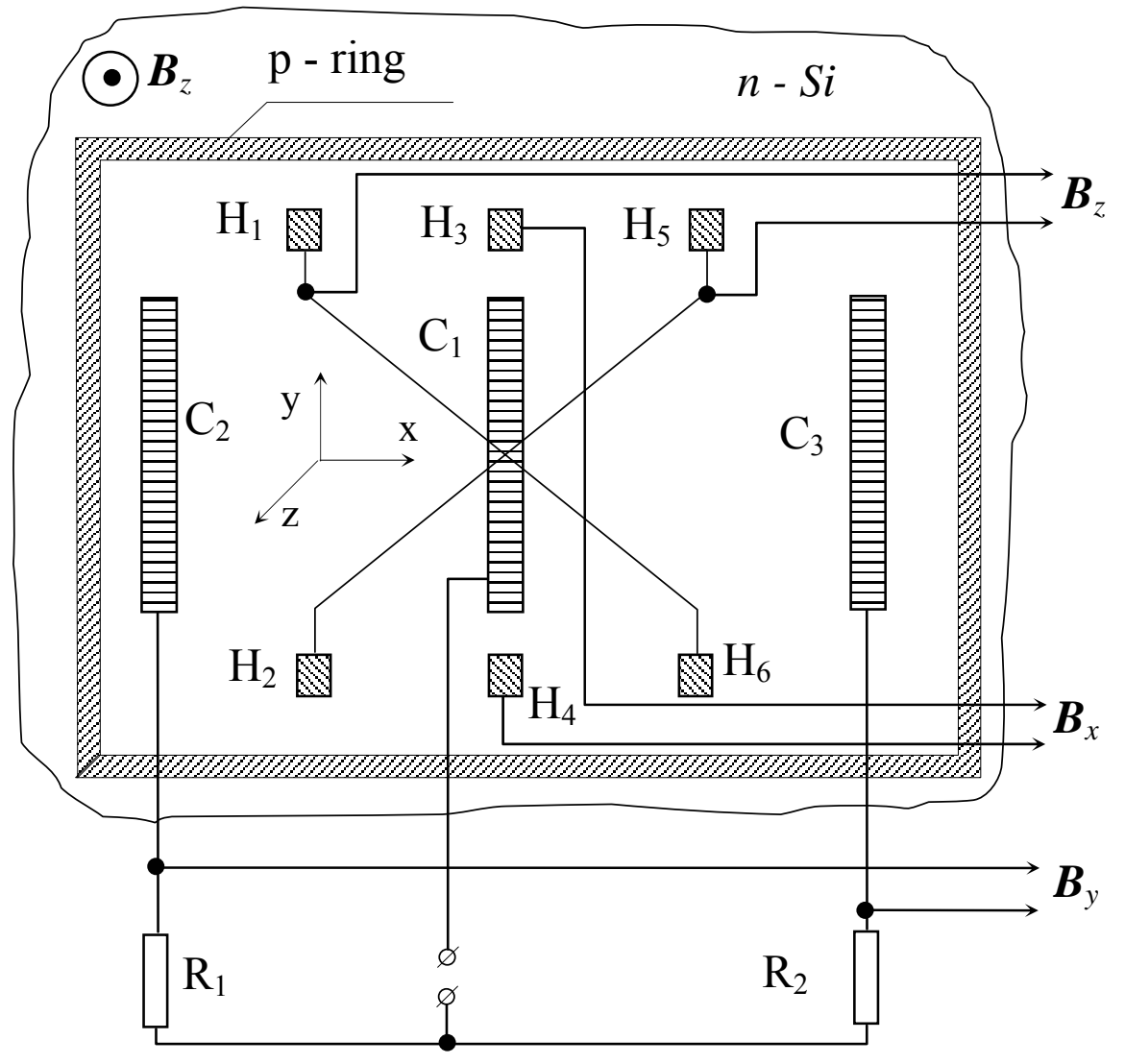

Fig. 6 - 3-D vertical Hall magnetometer for simultaneously detecting the $B_{x}, B_{y}$ and $B_{z}$ magnetic field components.

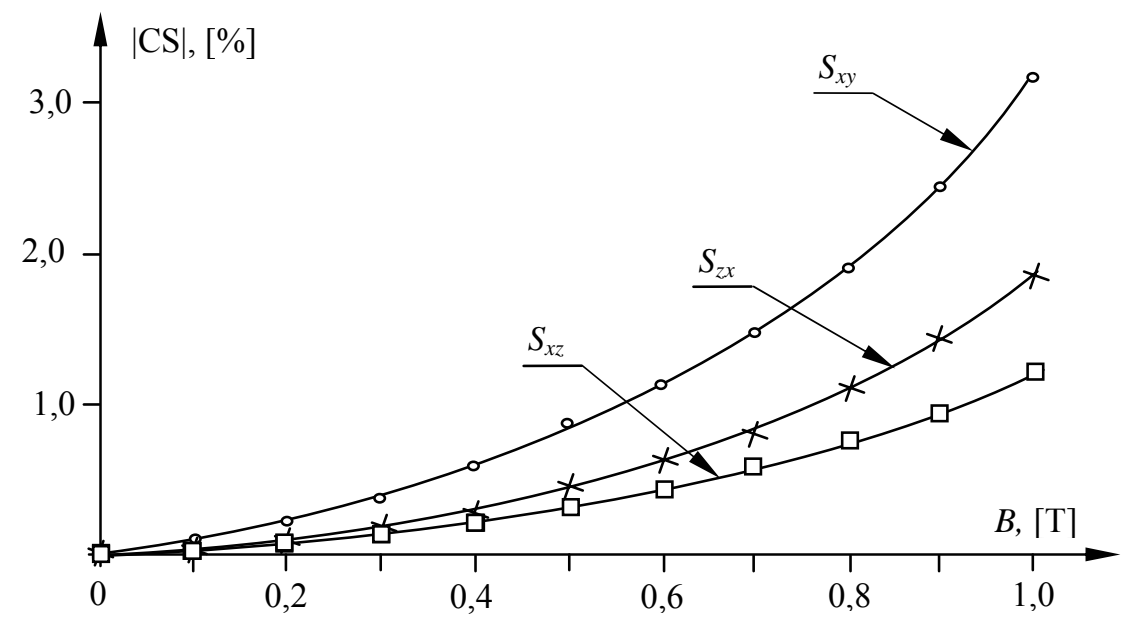

Fig. 7 - The cross-sensitivity between the $x$-, $y$-, and z- channels for $I_{\mathrm{C} 1}=10 \mathrm{~mA}, T=300$ 


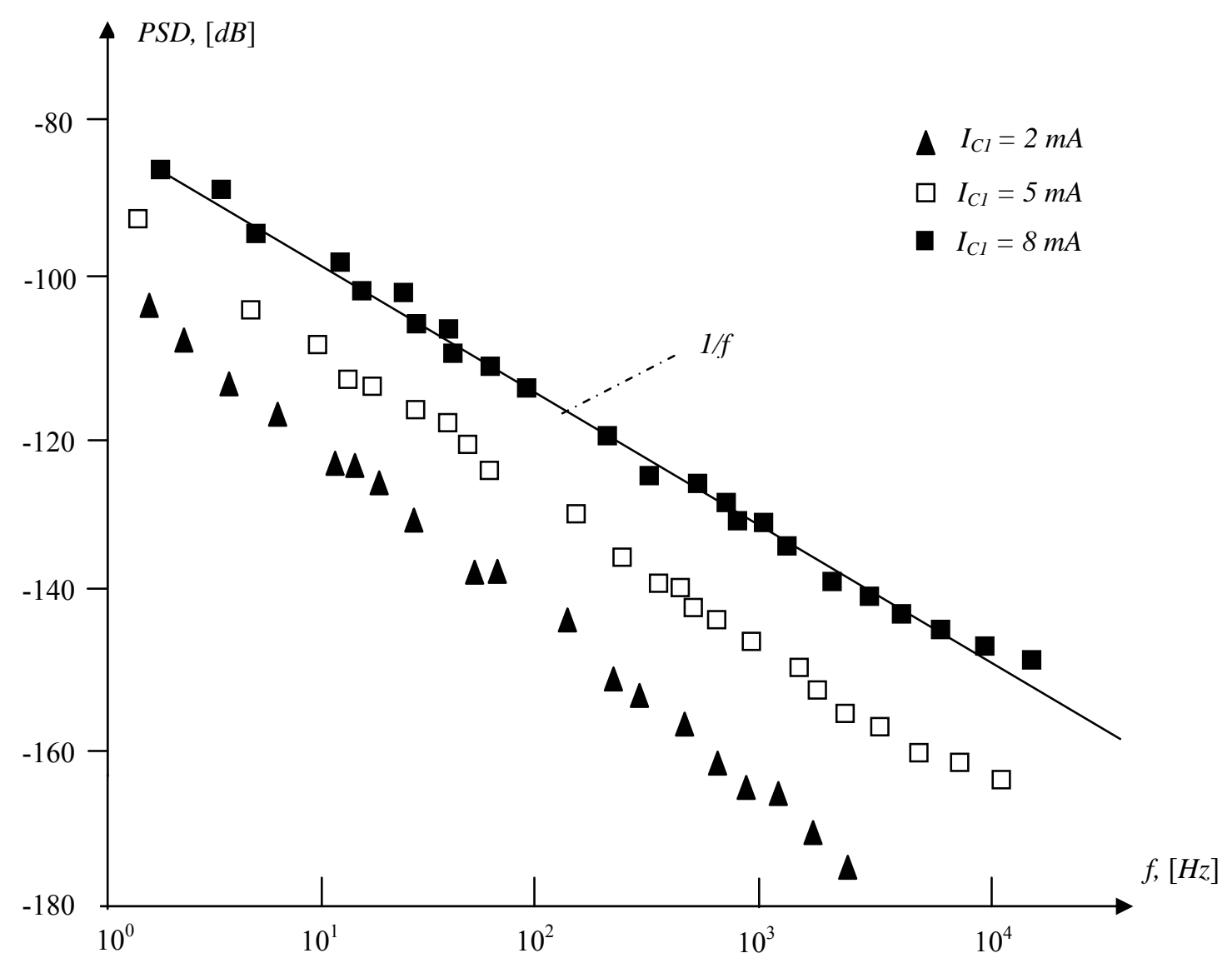

Fig. 8 - Noise power spectral density for one of the sensor channels. As a parameter is chosen the supply current $I_{C 1}$ at temperature $T=300 \mathrm{~K}$.

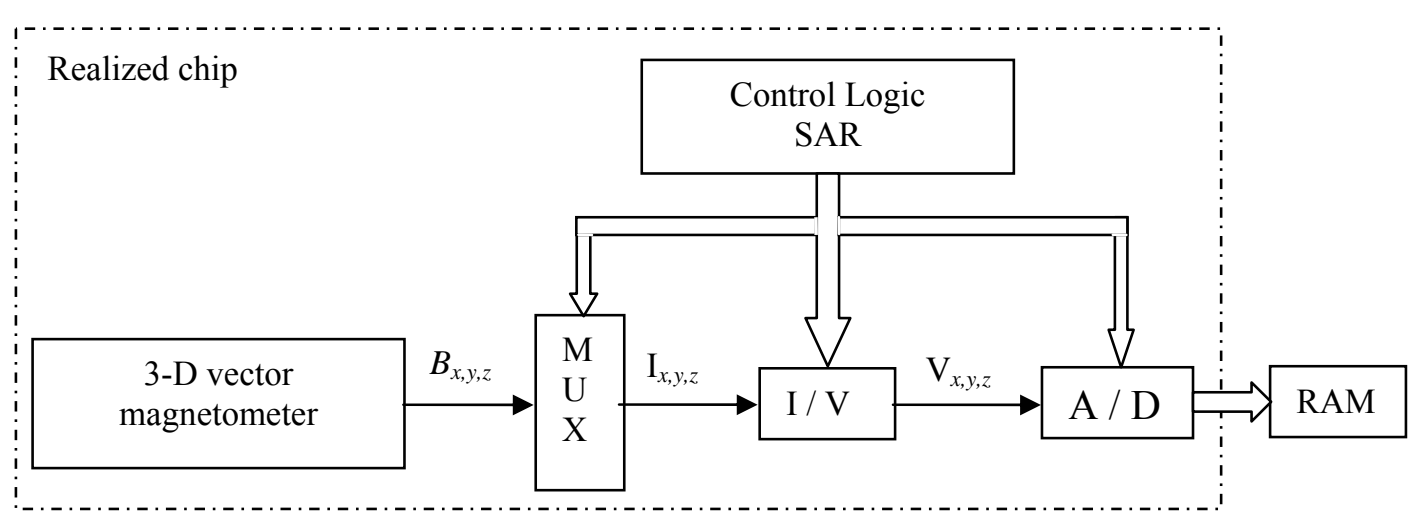

Fig. 9 - Block diagram of the integrated magnetodosimeter.

A few $\mathrm{mA}$ is too high to allow battery operation. This potential drawback is circumvented by choosing a particular architecture for the $\mathrm{A} / \mathrm{D}$ interface, which allows pulsed operation of the sensor with a very small duty cycle. Therefore, in spite of the large power consumption of the sensors, the average power consumption of the system is kept as low as $1 \mu \mathrm{W}$ from a $5 \mathrm{~V}$ power supply $[7,10]$.

The current signals produced by the sensor are multiplexed, transformed into voltages, equalized and converted into the digital domain using a single $\mathrm{A} / \mathrm{D}$ converter. The resulting words representing magnetic field components are delivered to the digital section, which calculates the module of the vector and stores the histogram of the magnetic field intensity during the monitored period into a RAM.

In light of the large current required to operate the magnetic sensor ( 2 to $10 \mathrm{~mA}$ ), we have to keep the sensor active only during a fraction of the acquisition cycle in order to fulfill the power 
consumption specifications. It is therefore necessary to use a low power sampling A/D converter, such as successive approximation architecture with two-step digital to analogue (D/A) converter.

\section{CONCLUSIONS}

The most important application area for Hall silicon sensors will undoubtedly be the smart sensors field. In the future, many large measurement and control systems will be bus oriented. In addition, smart Hall sensors will be a must in smart buildings and homes, smart traffic-control systems, smart cars etc. In this way of thoughts, improving and simplification of the $\mathrm{A} / \mathrm{D}$ conversion and the signal processing as a section of smart Hall systems will be essential part of the future work of the smart system designer scientists.

\section{ACKNOWLEDGMENT}

This research work was supported by the projects: "Micro and Nanotechnologies going Eastern Europe through Networking" (MINAEASTNET) within FP 6 (INCO - CT - 2004 - 510470) and "Multisensors", supported by Scientific Researches Fund (TH 1501 / 2005), Ministry of Education and Science of the Republic of Bulgaria. The presented results are useful for implementation in the area of smart magnetic sensors and Microsystems.

\section{REFERENCES}

[1] Ch. Roumenin, K. Dimitrov and P. Tzvetkov. Vertical Hall Effect Devices in the Basis of Smart Silicon Sensors. Proceedings of the Workshop "Intelligent Data Acquisition and Advanced Computing Systems: Technology and Applications (IDAACS'05)", Sofia, Bulgaria, 5-7 September 2005, pp. 55-58.

[2] H. Baltes, CMOS as Sensor Technology, Sensors and Actuators, A 37-38, 1993, pp. 51-56.

[3] S. Middelhock and S. A. Audet, Silicon Sensors, Academic Press, London, 1989.

[4] A. Haberli, M. Schneider, P. Malcovati, R. Castanetti, F. Maloberti and H. Baltes, "2-D Magnetic Microsensor with On-chip Signal Processing for Contactless Angle Measurements", Proc. of the IEEE Inter. SolidState Circuits Conf., Vol. 39, February 1996, pp. 332-333.

[5] H. Baltes, A. Haberli, P. Malcovati and F. Maloberti, "Smart Sensor Interfaces", ISCAS'96, Atlanta, Vol. 2, May 12-15, 1996, pp. 1380-1383.

[6] F. Maloberti, V. Liberali and P. Malcovati, Signal Processing for Smart Sensors, Proc. of
XI Brazilian Symp. of Integrated Circuit Design (SBCCI), Buzios (Rio de Jeneiro), Brazil, Sept./Oct. 1998, pp. 141-148.

[7] F. Maloberti and P. Malcovati, "Microsystems and Smart Sensors Interfaces: a Review", Journal of Analog Integrated Circuits and Signal Processing, Vol. 15, 1998, pp. 9-26.

[8] Ch. Roumenin, P. Kostov, Planar Hall sensor, Bulg. Patent № 37208, Dec. 26, 1983.

[9] Ch. Roumenin and K. Dimitrov, Microsystem for 2-D Magnetic-field Measurement, Journal of Lviv Polytechnic National University, Vol. 458, Publishing House of Lviv Polyt. National University, 2002, pp. 7-12.

[10] Ch. Roumenin, "Magnetic Vector Sensors based on the Hall Effect", C. R. Bulg. Sci., Vol. 42 (4), 1989, pp. 59-62.

[11] Ch. Roumenin, Solid State Magnetic Sensors, ELSEVIER, 1994.

[12] K. Dimitrov, "Smart Magnetic Sensors", Annual Proc. of the Technical University of Varna, Varna 7-9 October 2004, TU-Varna, Varna, Bulgaria, 2004, pp. 134-138.

[13] K. Dimitrov, "Magnetic Sensor Systems", Proc. of the $14^{\text {th }}$ Scientific Symp. Metrology and Metrology Assurance 2004, Sozopol 14-18 September 2004, Sozopol, Bulgaria, 2004, pp. 180-183.

[14] Ch. Roumenin, K. Dimitrov and A. Ivanov, "Novel Integrated 3-D Silicon Hall Magnetometer", Proc. of the EUROSENSORS XIV Conf., 27-30 August 2000, Copenhagen, Denmark, ISBN 87-89935-50-0, 2000, pp. 759761.

[15] Ch. Roumenin, K. Dimitrov and A. Ivanov, "Integrated Vector Sensor and Magnetic Compass using a novel 3-D Hall Structure", Sensors and Actuators, A 92, 2001, pp. 119122.

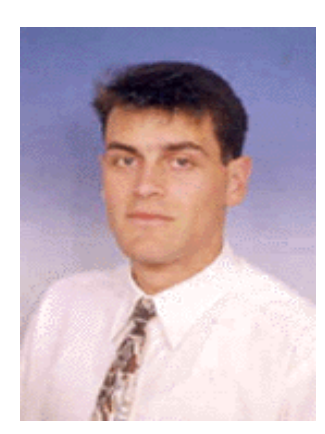

Konstantin V. Dimitrov is received his Ph.D. degree in measurement science from the Institute of Control and System Research (ICSR) with the Bulgarian Academy of Sciences (BAS), Sofia, Bulgaria (2002). Since July, 2002 he is Research Fellow at Sensor system and modeling Department at the ICSR. His research interests are focused on modeling and simulation of Hall effect devices, smart sensors and microsystems for magnetic field and temperature. 


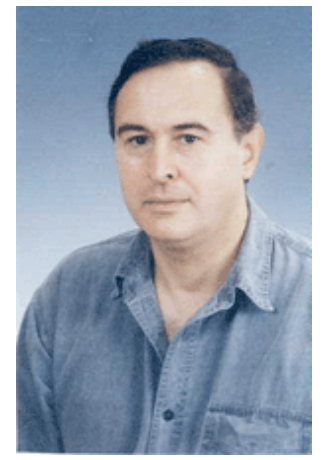

Chavdar S. Roumenin

received his M.S. degree in Semiconductor Electronics from Physics Dept. of Moscow State University (Russia) in January 1975. In 1977 he acquired his PhD degree on some peculiarities in $n$-InSb and their applications in magnetic-field transducers. Ch. Roumenin acquired his Doctor of Science degree in 1995 on the solid-state magnetic sensors and their applications. In 1996 he became a Professor, Department Head and Scientific Secretary in the
ICSR. In 2004 he is elected as Corresponding Member of the Bulagrian Academy of Sciences in the field of Engineer Sciences. Since 1999 he is a Director of ICSR. Prof. Ch. Roumenin is an author of more than 250 scientific papers, two invited books entitled "Solid State Magnetic Sensors" edited in 1994 by ELSEVIER SCl. and "Magnetic field microsensors" in MEMS Handbook by Andrew Publ. (2003), and 67 patents in the field of magnetic and thermal microsensors, microsystems, information technologies etc. He presented many times papers at EUROSENSORS Conferences and other International and regional activities. The articles of Ch. Roumenin have more than 1300 citations in international and national scientific journals. 